\title{
Digital Ischemia and Necrosis: A Rarely Described Complication of Gemcitabine in Pancreatic Adenocarcinoma
}

\author{
Eiichiro So, ${ }^{1}$ Zachary D. Crees, ${ }^{2, *}$ Danielle Crites, ${ }^{3}$ and Andrea Wang-Gillam ${ }^{3}$
}

\begin{abstract}
Background: Gemcitabine, alone or in combination with other agents, has become an important part of the standard of care for treatment of both resectable and unresectable/advanced pancreatic adenocarcinoma. Gemcitabine is generally considered to have a favorable toxicity profile, with myelosuppression and hepatotoxicity as the most common adverse effects. There are just two prior published case reports of gemcitabine-associated digital toxicity in the treatment of pancreatic adenocarcinoma, and few case reports when considering all solid tumors.

Presentation: A 70-year-old female developed hand numbness and tingling while receiving nab-paclitaxel plus gemcitabine for metastatic pancreatic adenocarcinoma. There was initial concern for Raynaud's or nab-paclitaxelassociated neuropathy, thus nab-paclitaxel was discontinued. However, her symptoms progressed to severe pain and her digits became dusky. An extensive evaluation revealed no alternative etiology except gemcitabineassociated digital ischemia (DI). The patient was treated with discontinuation of gemcitabine, and starting nitrates, opiates, calcium-channel blockers, and enoxaparin but eventually progressed to dry gangrene.

Conclusion: Here we report a case of gemcitabine-associated DI, along with a review of the literature. Although a rare complication, DI must be recognized and treated promptly to reduce the likelihood of serious and permanent morbidity.
\end{abstract}

Keywords: gemcitabine; digital ischemia; pancreatic adenocarcinoma; digital necrosis

\section{Introduction}

Gemcitabine as a single agent or in combination therapy is a mainstay treatment for pancreatic adenocarcinoma. ${ }^{1,2}$ Gemcitabine is generally well tolerated, with a favorable toxicity profile. ${ }^{1}$ There are case reports of gemcitabine-associated digital ischemia (DI), but there are only two prior case reports in the treatment of pancreatic adenocarcinoma. ${ }^{3-5}$ Here we present a case of gemcitabine-associated DI in a patient with pancreatic adenocarcinoma, along with a literature review.

\section{Case Report}

A 70-year-old female with metastatic pancreatic adenocarcinoma receiving gemcitabine and nab-paclitaxel presented to clinic with numbness and tingling in her bilateral hands. Owing to initial concern for nabpaclitaxel-associated neurotoxicity, nab-paclitaxel was discontinued while the patient was continued on gemcitabine (cumulative dose $4160 \mathrm{mg} / \mathrm{m}^{2}$ ). At 2-week follow-up, the patient reported progressive tenderness, coolness, and purple discoloration of her bilateral index fingers that was initially relieved with warm water immersion. Gemcitabine was promptly discontinued and the patient was admitted for further management. The patient had previously received FOLFOX and then FOLFIRI. She had a history of soleal deep vein thrombosis but no history of rheumatological, connective tissue or peripheral vascular disease. She was a 15-pack/

\footnotetext{
${ }^{1}$ Department of Medicine, Keio University School of Medicine, Tokyo, Japan.

${ }^{2}$ Department of Medicine, Washington University in Saint Louis, School of Medicine, Saint Louis, Missouri.

${ }^{3}$ Division of Hematology and Oncology, Department of Medicine, Washington University in Saint Louis, School of Medicine, Saint Louis, Missouri.
} 


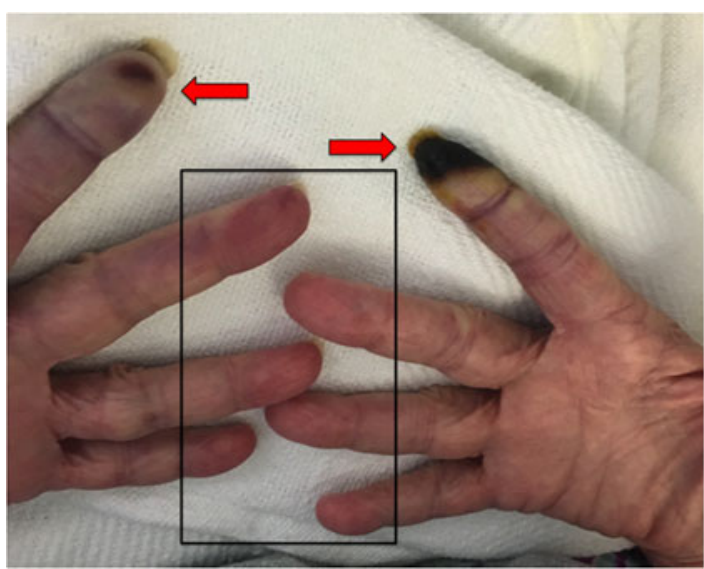

FIG. 1. Images of fingers/hands. Red arrows, bilateral index fingers demonstrating cyanosis, digital ischemia, and necrosis. Box, remaining digits demonstrating cyanosis and distal erythema. year smoker but quit 2 years previously. Her medications included diltiazem, pravastatin, and aspirin.

Upon admission, the patient's vital signs were within normal limits. Examination revealed bilateral necrotic index fingers (Fig. 1). Basic laboratories revealed leukocytosis, mild anemia, and hypoalbuminemia. The prothrombin time/international normalized ratio, partial thromboplastin time, lupus anticoagulant, anticardiolipin antibody, cryoglobulin S, C3/C4, and extractable nuclear antigen testing were unremarkable. Antinuclear antibody was positive with 1:320 titer and speckled pattern. Echocardiogram showed no evidence of intracardiac thrombi. CT angiography revealed no evidence of systemic emboli. Arterial Doppler studies showed immeasurable digit/arm indices (Fig. 2). Given the negative evaluation for competing etiologies, gemcitabineassociated DI was diagnosed. The patient was managed with gemcitabine discontinuation, along with initiation of calcium-channel blockade, nitrates, and opiates. Therapeutic enoxaparin was also initiated, but was subsequently discontinued because of gastrointestinal bleed. Despite treatment, the patient's symptoms progressed to dry gangrene of the bilateral index fingers.

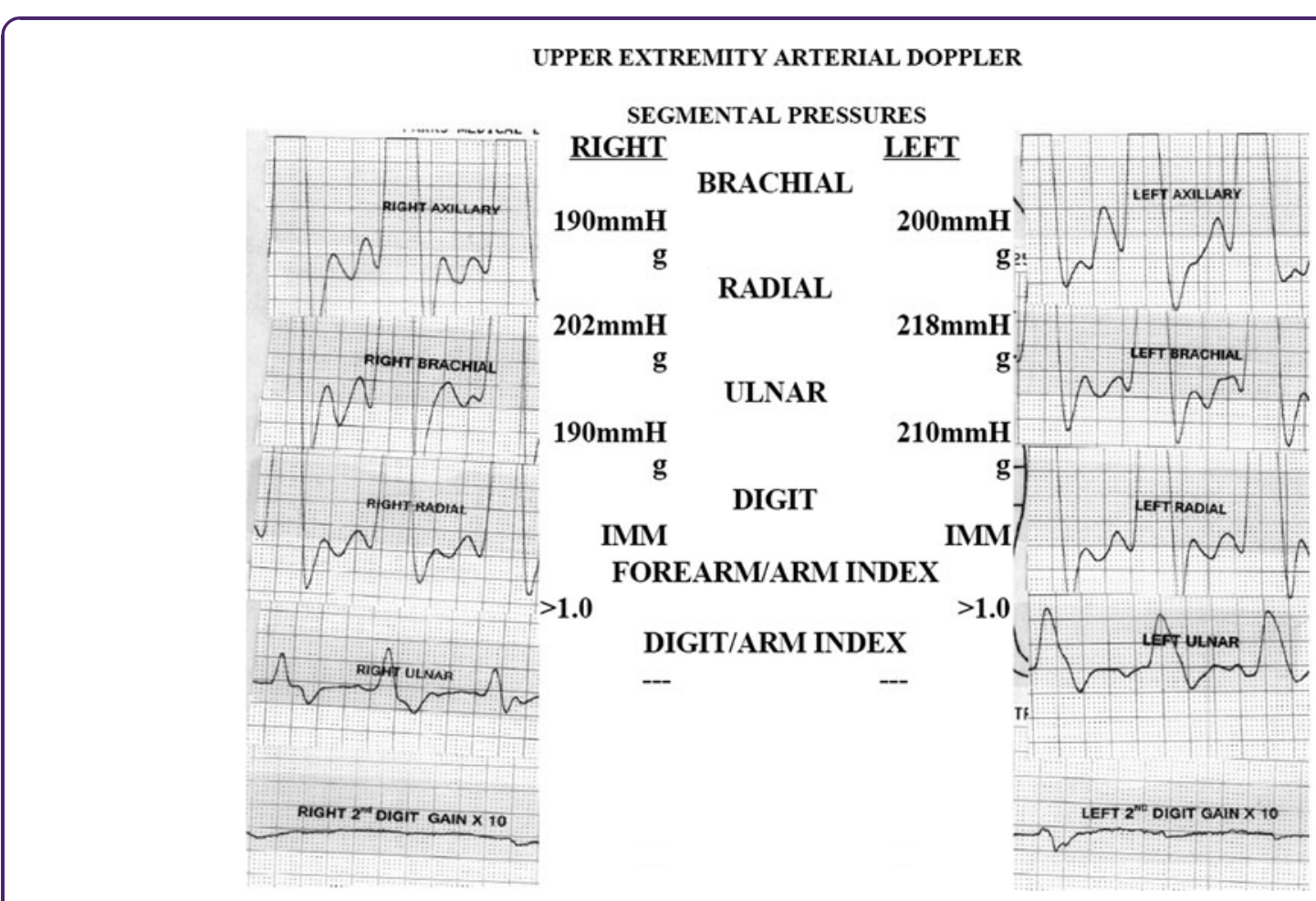

FIG. 2. Digit-arm indices. Digit-arm arterial Doppler tracings and indices demonstrating loss of arterial flow to the bilateral index fingers (second digit). 

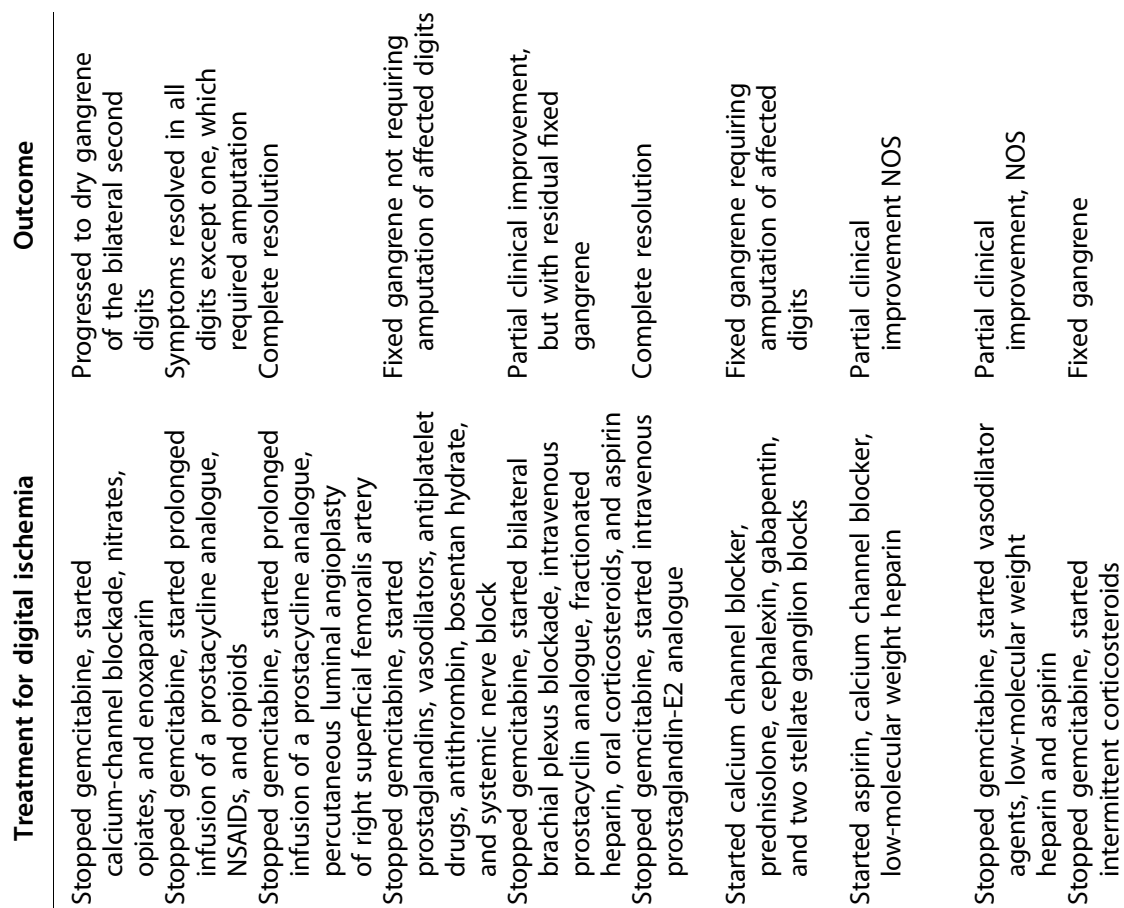

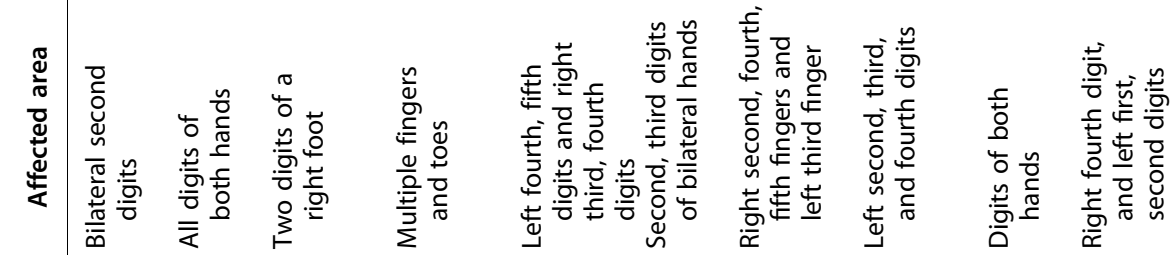

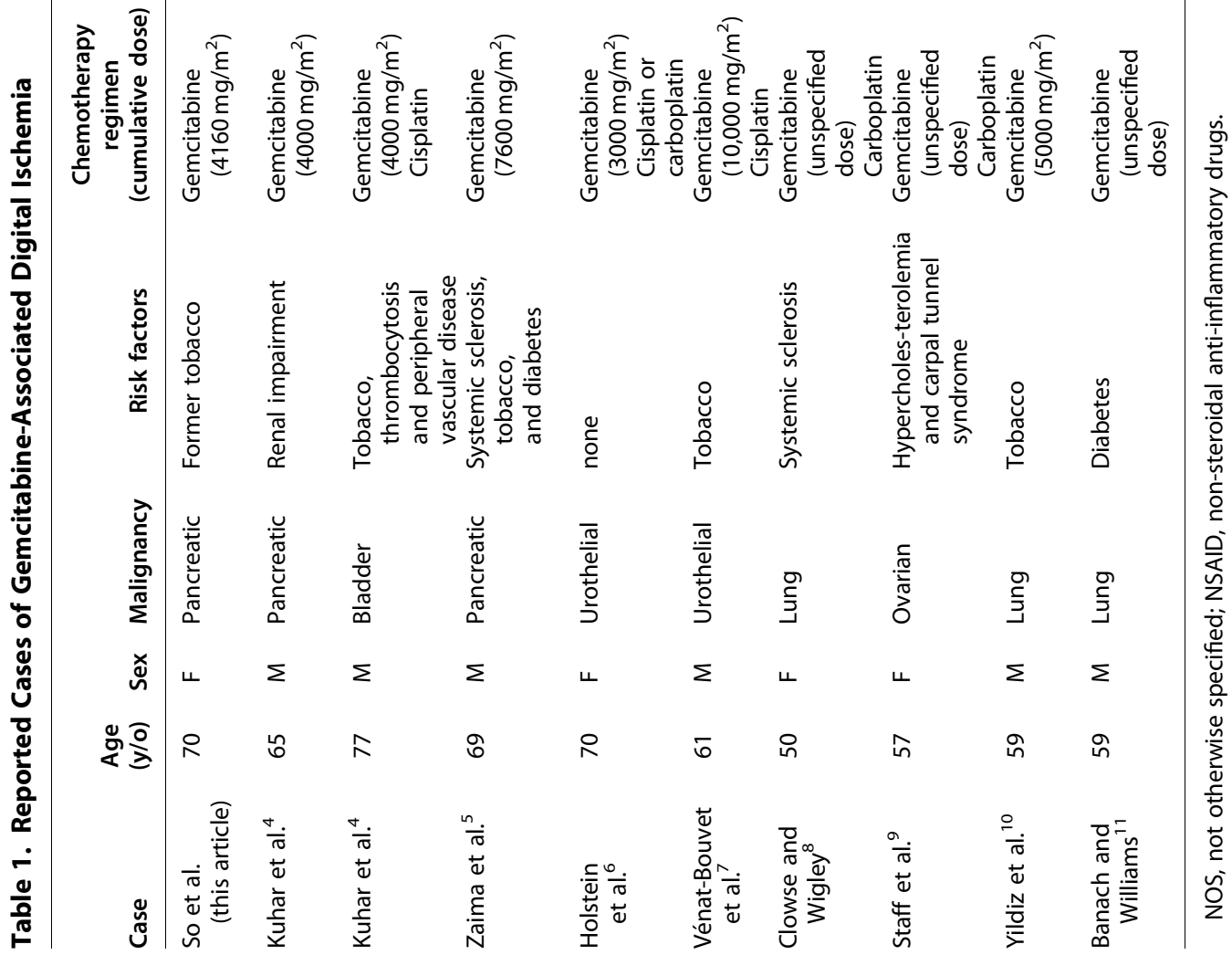




\section{Discussion}

Gemcitabine has been associated with thrombotic vascular complications affecting various organ systems. ${ }^{3}$ However, DI is a rare adverse effect of gemcitabine likely caused by drug-related microvascular endothelial damage and a relative hypercoagulable state, ${ }^{3}$ with only case reports to aid clinicians in identifying risk factors, recognizing signs/symptoms, and guiding management decisions. A PubMed search using "digital ischemia/ necrosis" and "gemcitabine," selecting English language articles and also using relevant cases from those articles' references revealed nine cases of gemcitabine-associated DI (Table 1), with only two cases occurring in pancreatic adenocarcinoma. Risk factors included tobacco use, diabetes, peripheral vascular disease, systemic sclerosis, combination chemotherapy, and cumulative gemcitabine dose $>10,000 \mathrm{mg} / \mathrm{m} .^{2,4-11}$ Two cases reported favorable outcomes. Kuhar et al. reported using infusion of a prostacycline analogue, NSAIDs, and opioids. ${ }^{4}$ Vénat-Bouvet et al. reported intravenous prostaglandin-E2 analogue alone. ${ }^{12}$ In each case, gemcitabine was withdrawn.

In this case, there was no underlying disease. Prior smoking was the only risk factor. The cumulative gemcitabine dose of $4160 \mathrm{mg} / \mathrm{m}^{2}$ was lower than previous reports. Given the lack of risk factors and initial suspicion for competing etiologies, gemcitabine was not immediately discontinued. Despite eventual discontinuation of gemcitabine and initiation of vasodilator therapy, the patient suffered irreversible digital necrosis. Therefore, based on variability in risk factors and cumulative dose exposure, clinicians should maintain a high degree of suspicion for gemcitabineassociated DI.

\section{Conclusion}

DI is a rare side effect of gemcitabine, which may be attributable to endothelial damage and a hypercoagulable state. There is variability in risk factors, presenting signs/symptoms, and treatment response, creating a diagnostic and treatment dilemma for clinicians. Withdrawing gemcitabine and initiation of prostaglandins and/or alternative vasodilators may be an effective treatment modality.

\section{Author Disclosure Statement}

Dr. Andrea Wang-Gillam serves on the advisory boards for Newlink, Pfizer, and Merrimack. The remaining authors have no relevant conflicts of interest or financial disclosures.

\section{References}

1. Burris HA, Moore MJ, Andersen J, et al. Improvements in survival and clinical benefit with gemcitabine as first-line therapy for patients with advanced pancreatic cancer: a randomized trial. J Clin Oncol. 1997;15:2403-2413.

2. Ottaiano A, Capozzi M, DeDivitiis C, et al. Gemcitabine monotherapy versus Gemcitabine plus targeted therapy in advanced pancreatic cancer: a meta-analysis of randomized phase III trials. Acta Oncologica. 2017:56:377-383.

3. Dasanu CA. Gemcitabine: vascular toxicity and prothrombotic potential. Expert Opin Drug Saf. 2008;7:703-716.

4. Kuhar CG, Mesti T, Zakotnik B. Digital ischemic events related to gemcitabine: Report of two cases and a systematic review. Radiol Oncol. 2010;44:257-261.

5. Zaima C, Kanai M, Ishikawa S, et al. A case of progressive digital ischemia after early withdrawal of gemcitabine and S- 1 in a patient with systemic sclerosis. Jpn J Clin Oncol. 2011;41:803-806.

6. Holstein A, Bätge R, Egberts EH. Gemcitabine induced digital ischaemia and necrosis. Eur J Cancer Care (Engl). 2010;19:408-409.

7. Vénat-Bouvet L, Ly K, Szelag JC, et al. Thrombotic microangiopathy and digital necrosis: two unrecognized toxicities of gemcitabine. Anticancer Drugs. 2003;14:829-832.

8. Clowse ME, Wigley FM. Digital necrosis related to carboplatin and gemcitabine therapy in systemic sclerosis. J Rheumatol. 2003;30:1341-1343.

9. Staff S, Lagerstedt E, Seppänen J, et al. Acute digital ischemia complicating gemcitabine and carboplatin combination chemotherapy for ovarian cancer. Acta Obstet Gynecol Scand. 2011;90:1296-1297.

10. Yildiz R, Coskun U, Kaya AO, et al. Digital ischemic changes after gemcitabine therapy in a patient with metastatic non-small-cell lung cancer. Ann Pharmacother. 2007;41:901-902.

11. Banach MJ, Williams GA. Purtscher retinopathy and necrotizing vasculitis with gemcitabine therapy. Arch Ophthalmol. 2000;118:726-727.

12. Vénat-Bouvet L, Ly K, Szelag JC, et al. Thrombotic microangiopathy and digital necrosis: two unrecognized toxicities of gemcitabine. Anticancer Drugs. 2003;14:829-832.

Cite this article as: So E, Crees ZD, Crites D, Wang-Gillam A (2017) Digital ischemia and necrosis: a rarely described complication of gemcitabine in pancreatic adenocarcinoma, Journal of Pancreatic Cancer 3:1, 49-52, DOI: 10.1089/pancan.2017.0012.

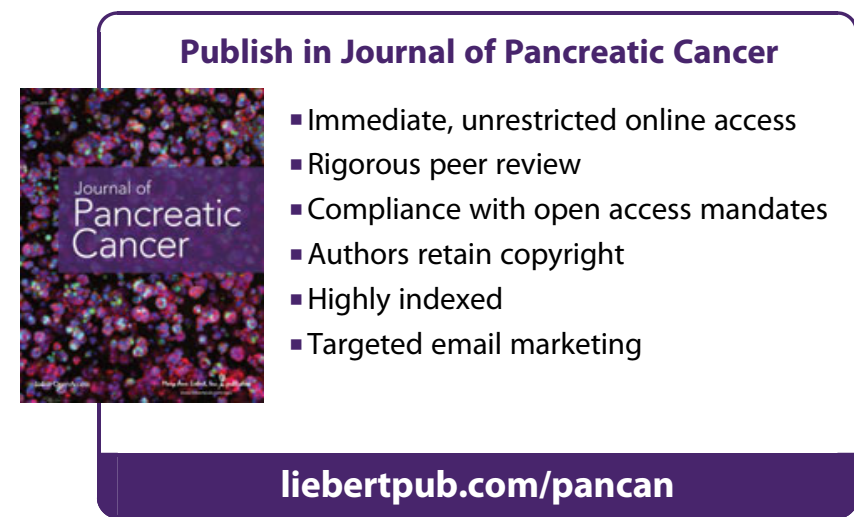

